\title{
Laboreal
}

Volume $17 \mathrm{~N}^{\circ} 1$ | 2021

Trabalhar hoje: mudanças, permanências, estratégias, reinvenções

\section{8 de abril, Día Mundial de la Seguridad y la Salud en el Trabajo: ¿qué aprender del COVID-19?}

28 de abril, Dia Mundial em Memória às Vitimas de Acidentes e Doenças do Trabalho: o que aprender com a COVID-19?

28 avril, Journée Mondiale de la Sécurité et de la Santé au Travail : que faut-il apprendre du COVID-19?

April 28, International Workers' Memorial Day: what to learn from COVID-19?

\section{Élida Azevedo Hennington}

Traductor: Fernanda Romero (fernandaromero.trad@gmail.com)

\section{OpenEdition}

\section{Journals}

Edición electrónica

URL: https://journals.openedition.org/laboreal/17668

DOI: 10.4000/laboreal. 17668

ISSN: 1646-5237

\section{Editor}

Universidade do Porto

\section{Referencia electrónica}

Élida Azevedo Hennington, «28 de abril, Día Mundial de la Seguridad y la Salud en el Trabajo: ¿qué aprender del COVID-19?», Laboreal [En línea], Volume 17 Nº1 | 2021, Publicado el 18 junio 2021, consultado el 20 junio 2021. URL: http://journals.openedition.org/laboreal/17668 ; DOI: https://doi.org/ 10.4000/laboreal.17668

\section{Este documento fue generado automáticamente el 20 junio 2021.}

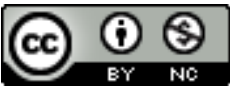

Laboreal está licenciado com uma Licença Creative Commons - Atribuição-NãoComercial 4.0 Internacional. 


\title{
28 de abril, Día Mundial de la Seguridad y la Salud en el Trabajo: ¿qué aprender del COVID-19?
}

\author{
28 de abril, Dia Mundial em Memória às Vítimas de Acidentes e Doenças do \\ Trabalho: o que aprender com a COVID-19? \\ 28 avril, Journée Mondiale de la Sécurité et de la Santé au Travail : que faut-il \\ apprendre du COVID-19? \\ April 28, International Workers' Memorial Day: what to learn from COVID-19?
}

\section{Élida Azevedo Hennington}

Tradución : Fernanda Romero (fernandaromero.trad@gmail.com)

1 En 2003, la Organización Internacional del Trabajo (OIT) reforzó la histórica actuación de los trabajadores y las entidades sindicales en defensa del trabajo decente, seguro y saludable, estableciendo el 28 de abril como el Día Mundial de la Seguridad y la Salud en el Trabajo, en memoria de las víctimas de accidentes y enfermedades profesionales (OIT, 2021). En ese día, se celebran eventos en todo el mundo para movilizar a los trabajadores, empleadores y gobiernos en torno al tema de los riesgos de accidentes y enfermedades relacionados con el trabajo, así como de sus graves consecuencias. El Día Nacional en Memoria de las Víctimas de Accidentes y Enfermedades Profesionales fue instituido en Brasil hace 16 años, con la Ley 11.121/2005.

2 En relación con la morbimortalidad profesional, las estimaciones de la OIT presentan datos alarmantes, como la ocurrencia anual de 2,3 millones de muertes en el mundo resultantes de accidentes o enfermedades profesionales, con aproximadamente 6.400 muertes diarias, según estas estadísticas (ILO, 2015).

Durante la conferencia internacional de 2019 de la OIT, la reunión anual que establece las normas internacionales del trabajo y las políticas generales de este organismo de las Naciones Unidas, los sindicatos reivindicaron el reconocimiento de que la salud y la seguridad en el trabajo deben ser incorporados en los principios y derechos fundamentales de la Organización: los derechos decentes universalmente aceptados y 
vinculantes que protegen a todos los trabajadores, en cualquier lugar. La Declaración del Centenario de la OIT reconoció que "las condiciones de trabajo seguras y saludables son fundamentales para el trabajo decente" (ILO, 2019).

4 Hoy en día, las enfermedades representan la principal causa de las muertes relacionadas con el trabajo, matando casi seis veces más trabajadores que los accidentes - los típicos y los de trayecto. Con relación a la morbilidad, se estima el surgimiento anual de 160 millones de casos de enfermedades profesionales en el mundo. Según la OIT, esto demuestra la necesidad de un nuevo paradigma de prevención: uno que también se centre en las enfermedades relacionadas con el trabajo y no solo en los accidentes (ILO, 2015).

La propagación del COVID-19 por todo el planeta y sus repercusiones en el mundo del trabajo, incluso después de la identificación y divulgación de los primeros casos todavía en 2019, parecen confirmar este nuevo paradigma.

\section{La pandemia de COVID-19 y el mundo del trabajo}

La pandemia del nuevo coronavirus resultó en una grave crisis humanitaria mundial, que afectó directamente a los trabajadores de todas las partes del mundo. Ha demostrado, de forma aguda, la creciente fragilidad tanto de los mecanismos de asistencia y promoción de la salud de los trabajadores, como de la prevención de accidentes y enfermedades relacionadas con el trabajo, evidenciando el progresivo deshilache de la red de protección social y de derechos. En este contexto, se produjo un fuerte clamor de los trabajadores durante el Congreso de la International Trade Union Confederation (ITUC, 2020) por la clasificación del COVID-19 como enfermedad profesional.

7 La falta de protección de la salud de los trabajadores, expuesta dramáticamente por la pandemia en varias partes del mundo, dictó el tema de la campaña de 2021: "La salud y la seguridad son un derecho fundamental en el trabajo". Según la OIT, se consideran derechos fundamentales y con el mayor grado de compromiso y responsabilidad de los gobiernos: la libertad de asociación y el derecho a la negociación colectiva, la eliminación del trabajo forzoso y del trabajo infantil y el fin de la discriminación en el empleo/ocupación. Las entidades sindicales piden la incorporación de otro derecho que refuerce la necesidad de una protección efectiva en el lugar de trabajo y la atención a la salud de los trabajadores (ITUC, 2021).

8 Parece, entonces, que la evolución de la pandemia en el mundo no hizo más que reforzar el motivo por el cual la salud y la seguridad se deben consolidar como un derecho de todos los que trabajan. Y, de hecho, la aparición de brotes de COVID-19 que afectan a diferentes categorías profesionales y en distintos procesos y lugares de trabajo, así como el creciente número de enfermedades y muertes de trabajadores de primera línea en la atención a la población, considerados "esenciales", encendieron la señal de alarma.

En verdad, la demora en brindar buenas condiciones de trabajo, equipos de protección adecuados y suficientes y en promover el registro de estos hechos como accidentes de trabajo, o incluso en reconocer los derechos de estos trabajadores y de sus familias, no hizo más que confirmar, de manera grave, abrupta y a gran escala, la paulatina y creciente transformación y crisis en el mundo del trabajo, agravadas por las políticas 
neoliberales, en un proceso incesante de precarización y pérdida de derechos a lo largo del tiempo.

10 La evolución de la pandemia ha llevado a gobiernos, empleadores, trabajadores y a la población en general a enfrentarse a retos sin precedentes. Así, en lo que respecta a la OIT, se decidió que el Día Mundial de la Seguridad y la Salud en el Trabajo se centrará en estrategias para fortalecer los sistemas nacionales de seguridad y salud en el sentido de construir resiliencia ante las adversidades actuales y futuras, con base en las experiencias vividas y las lecciones aprendidas con el COVID-19 (OIT, 2020).

Muchas entidades sindicales, investigadores e instituciones de defensa de los derechos colectivos e individuales en el ámbito laboral han señalado la necesidad de resguardar la salud y garantizar los derechos a los trabajadores expuestos al riesgo de infección, enfermedad y muerte por el nuevo coronavirus, bien como asegurar la protección de sus familias (Alici et al., 2020; Carlsten, 2021; George \& George, 2020; Moen, 2020). La prevención y la puesta en práctica de políticas compensatorias y de protección de la salud son pasos importantes en la lucha de los trabajadores por unas mejores condiciones de vida y de trabajo.

\section{COVID-19 y el reconocimiento de su relación con el trabajo}

Según la ISSA (International Social Security Association) - entidad creada en 1927 bajo los auspicios de la OIT y que hoy reúne a más de 300 organizaciones de seguridad social en más de 150 países -, si bien es cierto que el COVID-19 aún no está incluido en la lista de enfermedades profesionales de la OIT, como la hepatitis B y C y el VIH, sí puede clasificarse como enfermedad profesional causada por un agente biológico. Esto, siempre que exista una relación directa establecida científicamente o determinada por métodos adecuados entre la exposición al agente biológico, la actividad de trabajo y la enfermedad (ISSA, 2020). Las evidencias científicas de que el SARS-Cov-2 causa el COVID-19 ya están establecidas, pero, en muchos casos, es difícil probar la exposición laboral o que la enfermedad se contrajo en el decurso del trabajo.

13 A medida que avanzaba la pandemia, países como Noruega, Italia, Alemania, Canadá, Francia, EE.UU. y Sudáfrica reconocieron la enfermedad causada por el nuevo coronavirus como profesional, con diferencias en la caracterización y considerando como categorías afectadas especialmente a médicos, enfermeros y otros profesionales de la salud (Chirico \& Magnavita, 2020; Godderis et al., 2020; ISSA, 2020; OSHA, 2020/2021).

El médico italiano Antonio Mutti ha incluso afirmado que el COVID-19 representa una nueva enfermedad profesional, la más mortífera de los últimos tiempos (Mutti, 2020). Cabe señalar que, desde el primer brote en Wuhan, la mayoría de los casos fueron de origen profesional, afectando principalmente a las personas que trabajaban en el mercado mayorista de mariscos y otros animales. Posteriormente, los profesionales de la salud se convirtieron en un grupo de alto riesgo: del total de 138 pacientes tratados en un hospital de Wuhan, 40 pacientes (el 29\% de los casos) eran profesionales de la salud, 31 de los cuales (el 77,5\%) trabajaban en enfermerías generales, 7 (el 17,5\%) en urgencias y 2 (el 5\%) en la unidad de cuidados intensivos (UCI). 

pandemia están claramente más expuestos, y no sólo al peligro de infección por el nuevo coronavirus, a la muerte, a la enfermedad y a las posibles secuelas, sino también a otros daños a la salud, como la fatiga por las largas jornadas de trabajo, el sufrimiento psicológico, el síndrome de burnout y diversos problemas de salud mental debido al estrés crónico/experiencias traumáticas, estigma y violencia (Godderis et al., 2020; Mutti, 2020; WHO, 2020). Tal y como han comprobado Burdorf et al. (2020), el COVID-19 constituye un enorme reto, ya que trabajadores de muchas ocupaciones distintas a la de los profesionales de salud se enfrentan a un alto riesgo de exposición al virus. Hay, de hecho, una larga lista de ocupaciones que implican un contacto directo con el público y la proximidad física con otras personas, como los barberos, los manicuros, los trabajadores del comercio, de tiendas, bares, restaurantes y servicios de reparto, etc. Por otro lado, hay actividades en las que los trabajadores, al contrario de los considerados legalmente como "esenciales", tienen la libertad de trabajar desde casa, lo que reduce considerablemente el riesgo de infección.

En Italia, a raíz de los debates sobre esta materia, han emergido interesantes hipótesis. El estudio de Marinaccio et al. (2020), al investigar los pedidos de indemnización de los trabajadores de ese país debido a la infección por el nuevo coronavirus, identificó que el COVID-19 había sido adquirido en el lugar de trabajo en una proporción considerable de los casos (el 19,4\%). Los principales sectores económicos afectados fueron las actividades relacionadas con la salud humana y el servicio social, aunque las reivindicaciones de compensación profesional también incluyeron a otros trabajadores de sectores esenciales, como plantas de procesamiento de carne y aves, sectores de almacenamiento, empleados de tiendas, carteros, farmacéuticos y trabajadores de limpieza. El estudio concluyó que es necesario construir un sistema de vigilancia de la salud de los trabajadores para los casos de COVID-19 que incluya un análisis individualizado de las circunstancias en las que se adquiere la infección, para la prevención de riesgos laborales, la orientación y el apoyo al sistema de seguros y la gestión de la política de vacunación.

Esto es especialmente relevante en el caso de las enfermedades profesionales emergentes, como el COVID-19, cuando los conocimientos sobre la enfermedad y los factores de riesgo asociados son limitados y la aplicación de medidas de prevención es urgente. Van der Molen et al. (2020) señalan que, en los Países Bajos, aunque los pedidos de indemnización puedan estar influenciados por la necesidad económica del trabajador y el registro de las enfermedades profesionales pueda estar sesgado por la actuación de los médicos del trabajo, la notificación y el análisis de la información sobre los casos de COVID-19 relacionados con el trabajo son importantes para identificar a los grupos más vulnerables y explorar el impacto de los factores de riesgo.

Parece evidente que los trabajadores -en especial los llamados trabajadores esencialesestán muy expuestos al SRAS-CoV-2, más que la población en general. Un equipo de investigadores canadienses (Carlsten et al., 2021) destacó algunos puntos relevantes para la protección de los trabajadores que actúan en el enfrentamiento a la pandemia: factores como el bajo nivel socioeconómico confieren una desventaja y aumentan la susceptibilidad y vulnerabilidad de estos trabajadores, agravando el impacto del COVID-19, ya que, en este caso, el contacto con el virus es prácticamente inevitable y la aplicación de los fundamentos de la seguridad y salud laboral son críticos.

Laboreal, Volume $17 \mathrm{~N}^{\circ} 1$ | 2021 
19 Sería imprescindible proporcionar un entorno de trabajo seguro y saludable, con el máximo cuidado y la protección adecuada de los trabajadores, asegurándoles medidas de higiene y prevención de la contaminación, como la realización de pruebas a los trabajadores más expuestos, vulnerables y susceptibles; la puesta en marcha de planes de salud y seguridad para lugares y puestos de trabajo específicos, con la provisión de equipos de protección individual y colectiva; la adopción de medidas para identificar y aislar los casos de infección; el desarrollo de sistemas de información y vigilancia sanitaria; y la difusión pública de datos e información. Los autores finalizan afirmando que, a partir de ahora, tenemos que estar preparados para otros eventos catastróficos como la pandemia del Covid-19 y no debemos subestimar los riesgos de nuevas catástrofes mundiales (Carlsten et al., 2021).

En el caso de Italia, el Instituto Nacional del Seguro de Accidentes de Trabajo (INAIL Istituto Nazionale Assicurazione Infortuni sul Lavoro) ha afirmado recientemente que es indispensable reconocer el COVID-19 como enfermedad relacionada con el trabajo, no sólo en lo que se refiere a los médicos y enfermeros, sino también a otros trabajadores de primera línea, como los que actúan en contacto directo con el público (por ejemplo, asistentes sociales y empleados de hospitales, cajeros de supermercados y otros trabajadores comerciales, entre otros). En el caso de este grupo, existe en Italia una presunción legal de enfermedad profesional, lo que puede permitir la concesión inmediata de prestaciones de seguro. En otras situaciones, todavía, la relación de causa y efecto debe ser demostrada caso por caso por el trabajador que solicita la indemnización. Sin embargo, hay que subrayar que, dada la presencia del virus no sólo en el entorno laboral, sino también fuera de él, demostrar su origen profesional puede ser imposible para el trabajador (probatio diabolica, en latín), y el correcto encuadramiento del COVID-19 como enfermedad relacionada con el trabajo se convierte en un verdadero reto (Chirico \& Magnavita, 2020).

\section{COVID-19 y la salud de los trabajadores en Brasil}

Moen (2020) buscó señalar, en un editorial de la revista Occupational Medicine, la importancia del reconocimiento mundial de la enfermedad causada por el nuevo coronavirus como profesional, con el fin de posibilitar compensaciones, generar estudios y análisis estadísticos y permitir la planificación de medidas preventivas en materia de salud y seguridad.

En Brasil, al igual que en otras partes del mundo, hay un movimiento en defensa del reconocimiento del COVID-19 como enfermedad relacionada con el trabajo en el caso de los trabajadores que se vieron obligados a realizar sus actividades laborales fuera de sus hogares (Maeno \& Carmo, 2020). Los argumentos se referían, inicialmente, a las situaciones cotidianas de exposición al virus y a la facilidad de diseminación de la infección, especialmente por parte de personas asintomáticas u oligosintomáticas. A este cuadro se suma la falta de condiciones de trabajo, de estructura y de políticas sociales e de control de la pandemia en el país, la incapacidad de brindar medidas de aislamiento y distanciamiento social, la exposición forzada al riesgo en los ambientes de trabajo, en los transportes y en las vías públicas, la imposibilidad de ejercer el derecho legal a rechazar trabajos considerados de riesgo grave o inminente, entre otras limitaciones. 

pandemia y permanece como determinante fundamental del proceso saludenfermedad. Más que nunca, los trabajadores que sostienen la economía y el desarrollo de los países tienen que luchar y estar unidos en la defensa de la vida, en la búsqueda de mejores condiciones de trabajo, de una renta básica y de más empleos, en el rescate y la conquista de los derechos laborales y sociales, en definitiva, de una vida sana y digna. 


\section{BIBLIOGRAFÍA}

Alici, N., Beyan, A., \& Simsek, C. (2020). COVID-19 as an occupational disease. Eurasian Journal of Pulmonology, 22(4), 90-100. https://doi.org/10.4103/ejop.ejop_50_20

Burdorf, A., Porru, F., \& Rugulies, R. (2020). The COVID-19 (Coronavirus) pandemic: consequences for occupational health. Scandinavian Journal of Work, Environment \& Health, 46(3), 229-230. https:// doi.org/10.5271/sjweh.3893

Carlsten, C., Gulati, M., Hines, S., Rose, C., Scott, K., Tarlo, S. M., Torén, K., Sood, A., \& de la Hoz, R. (2021). COVID-19 as an occupational disease. American Journal of Industrial Medicine, 64(4), 227-237. https://doi.org/10.1002/ajim.23222

Chirico, F., \& Magnavita, N. (2020). COVID-19 infection in Italy: an occupational injury. South African Medical Journal, 110(6), 436. https://doi.org/10.7196/SAMJ.2020.v110i6.14855

George, R., \& George, A. (2020). COVID-19 as an occupational disease? South African Medical Journal, 110(4), 260. https://doi.org/10.7196/SAMJ.2020.v110i4.14712

Godderis, L., Boone, A., \& Bakusic, J. (2020). COVID-19: a new work-related disease threatening healthcare workers. Occupational Medicine, 70(5), 315-316. https://doi.org/10.1093/occmed/ kqaa056

International Labour Organization - ILO (2015). Global trends on occupational accidents and diseases. Available on https://www.ilo.org/legacy/english/osh/en/story_content/external_files/fs_st_1ILO_5_en.pdf

International Labour Organization - ILO (2019). Centenary declaration for the future of work adopted by the conference at its one hundred and eighth session. Available on https://www.ilo.org/wcmsp5/ groups/public/@ed_norm/@relconf/documents/meetingdocument/wcms_711674.pdf

International Social Security Association - ISSA (2020, April 2). Can COVID-19 be considered an occupational disease? Available on https://ww1.issa.int/analysis/can-covid-19-be-consideredoccupational-disease

International Trade Union Confederation - ITUC (2020, April 27). COVID-19 should be classified as an occupational disease. Available on https://www.ituc-csi.org/covid-19-occupational-disease? lang=en

International Trade Union Confederation - ITUC (2021, February 4). Save lives at work: International Workers' Memorial Day - 28 April. Available on https://www.ituc-csi.org/save-lives-at-work? lang=en

Maeno, M., \& Carmo, J. C. (2020, May 17). A COVID-19 é uma doença relacionada ao trabalho. Observatório da Medicina. Disponível em http://observatoriodamedicina.ensp.fiocruz.br/acovid-19-e-uma-doenca-relacionada-ao-trabalho-por-maria-maeno-e-jose-carlos-do-carmo

Marinaccio, A., Boccuni, F., Rondinone, B., Brusco, A., D’Amario, S., \& Iavicoli, S. (2020). Occupational factors in the COVID-19 pandemic in Italy: compensation claims applications support establishing an occupational surveillance system Occupational and Environmental Medicine, 77(12), 818-821. https://doi.org/10.1136/oemed-2020-106844

Ministério da Economia (2020). Nota Técnica SEI nº 56376/2020/ME. COVID-19. Nexo com o trabalho à luz da legislação Previdenciária. Medida Provisória $n^{\circ}$ 927, de 2020. Secretaria Especial de Previdência e Trabalho, Secretaria de Previdência, Subsecretaria do Regime Geral de Previdência Social, 
Coordenação-Geral de Benefícios de Risco e Reabilitação Profissional. https://www.gov.br/ economia/pt-br/centrais-de-conteudo/publicacoes/notas-tecnicas/2020/sei_me-12415081-notatecnica-covid-ocupacional.pdf

Moen, B. (2020). COVID-19 should be recognized as an occupational disease worldwide. Occupational Medicine, 70(5), 299. https://doi.org/10.1093/occmed/kqaa086

Mutti, A. (2020). Occupational Medicine in the time of COVID-19. La Medicina del Lavoro, 111(2), 83-86. https://doi.org/10.23749/mdl.v111i2.9546

Occupational Safety and Health Administration - OSHA (2020/2021). COVID-19 Regulations. United States Department of Labor. https://www.osha.gov/coronavirus/standards

Organização Internacional do Trabalho - OIT (2014). Nota Informativa - Dia Mundial de Segurança e Saúde no Trabalho. https://www.ilo.org/legacy/english/protection/safework/worldday/ products04/factsheet04_por.pdf

Organização Internacional do Trabalho - OIT (2020). Frente a la pandemia: garantizar la seguridad y salud en el trabajo. https://www.ilo.org/wcmsp5/groups/public/---ed_protect/---protrav/--safework/documents/publication/wcms_742732.pdf

Van der Molen, H. F., Kezic, S., Visser, S., de Groene, G., Maas, J., de Wind, A., \& Tamminga, S. (2020). COVID-19: what can be learned from notifications of occupational diseases? Occupational and Environmental Medicine, 78(6), 464. https://doi.org/10.1136/oemed-2020-107121

World Health Organization - WHO (2020). Coronavirus disease (COVID-19) outbreak: rights, roles and responsibilities of health workers, including key considerations for occupational safety and health. Available on https://www.who.int/docs/default-source/coronaviruse/who-rights-roles-responhw-covid-19.pdf?sfvrsn=bcabd401_0

\section{AUTORES}

\section{ÉLIDA AZEVEDO HENNINGTON}

http://orcid.org/0000-0001-5280-8827

Centro de Estudos da Saúde do Trabalhador e Ecologia Humana, Escola Nacional de Saúde Pública Sergio Arouca, Fundação Oswaldo Cruz, Rio de Janeiro, Brasil, Rua Leopoldo Bulhões, 1480 -

Manguinhos, Rio de Janeiro. CEP 21040-360

elida.hennington@ensp.fiocruz.br 